\title{
An OFDM System Concept for Joint Radar and Communications Operations
}

\author{
Christian Sturm, Thomas Zwick, and Werner Wiesbeck \\ Institut für Hochfrequenztechnik und Elektronik, University of Karlsruhe (TH) \\ Kaiserstraße 12, 76131 Karlsruhe, Germany \\ christian.sturm@ihe.uka.de
}

\begin{abstract}
In this paper the possibility of designing an OFDM system for simultaneous radar and communications operations is discussed. A novel approach to OFDM radar processing is introduced that overcomes the typical drawbacks of correlation based processing. A suitable OFDM system parameterization for operation at $24 \mathrm{GHz}$ is derived that fulfills the requirements for both applications. The operability of the proposed system concept is verified with MatLab simulations.
\end{abstract}

Keywords-OFDM; Radar, Communications

\section{INTRODUCTION}

Even though the idea of combining radar and communications in one single system platform has been existing for a long time [1], there has never been introduced any relevant implementation of such concept. A joint radar and communications system would constitute a unique costefficient platform for future intelligent transportation systems, for which both environment sensing and the allocation of adhoc communication links are essential tasks. The most important reason for not introducing such systems might be because traditional radar platforms and signals have been too different from their communications counterparts. Nowadays, when the baseband processing can be easily performed in the digital domain, these restrictions do not apply any longer. Also as a result of this progress, in recent years orthogonal frequency division multiplexing (OFDM) in combination with phase shift keying (PSK) has become a popular modulation technique in new communications standards. OFDM offers several advantages like robustness against multi-path fading, easy synchronization and equalization, and a high flexibility in system design, which allows for easily adapting the system parameters to the given channel characteristics. Furthermore, it has been shown that OFDM-like signals are also suitable for radar applications [2]. Also, it has already been proposed in literature to implement radar networks with integrated communications functions based on OFDM signals [3].

In this paper the feasibility of the design of an OFDM system that even simultaneously allows for performing radar and communications operations is investigated. First, the OFDM signal structure is regarded and an already existing approach for OFDM radar processing is compared to a novel one that is proposed later in this paper. Requirements for the system parameterization from both radar and communications perspective are analyzed. A parameterization for operation in the $24 \mathrm{GHz}$ ISM band that allows for simultaneous operation of both functions is derived. Finally, simulation results for the novel processing approach from a MatLab model are presented.

\section{OFDM RADAR}

Strictly speaking OFDM is not a modulation technique but a multiplexing technique, which allows for multiplexing the transmission of symbols over orthogonal subcarriers. These subcarriers are defined by basis functions

$\psi_{n}(t)=\exp \left(j 2 \pi f_{n} t\right) \frac{1}{\sqrt{T}} \operatorname{rect}\left(\frac{t}{T}\right), \quad n=0, \ldots, N-1$

with $f_{n}$ representing the individual subcarrier frequencies, $T$ denoting the OFDM symbol duration, and $N$ being the number of subcarriers. These basis functions are orthogonal when

$$
\Delta f=\frac{1}{T}
$$

hence, this spacing has to be chosen in order to avoid intercarrier interference. With this choice we obtain for the individual subcarrier frequencies

$$
f_{n}=f_{\min }+n \Delta f, \quad n=0, \ldots, N-1
$$

with $f_{\min }$ being an arbitrary frequency. An arbitrary information series $\{I(n)\}$ consisting of complex modulation symbols obtained through discrete phase modulation (e.g. PSK) is converted to an OFDM signal by projecting the modulation symbols onto the orthogonal basis functions. The resulting time domain signal can be expressed as

$$
x(t)=\sum_{\mu=-\infty}^{\infty} \sum_{n=0}^{N-1} I(\mu N+n) \psi_{n}(t-\mu T)
$$


where $\mu$ represents the time index of the subsequent OFDM symbols. From this result one single OFDM-symbol can be extracted by regarding only $\mu=0$, which results in

$$
x(t)=\sum_{n=0}^{N-1} I(n) \exp \left(j 2 \pi f_{n} t\right), \quad 0 \leq t \leq T
$$

In the frequency domain this corresponds to

$$
X(f)=\sum_{n=0}^{N-1} I(n) \sqrt{T} \frac{\sin \left(\pi\left(f-f_{n}\right) T\right)}{\left(\pi\left(f-f_{n}\right) T\right)}
$$

Hence, the resulting spectrum is the sum of $N$ sinc-functions, each shifted with constant spacing $\Delta f$ on the frequency axis.

The OFDM radar platform is supposed to be realized as a monostatic system and hence will be equipped with one transmitter and one receiver. The radiated signal, which is intended to transmit information to a distant receiver, will at the same time be scattered from objects in the neighborhood of the platform and also the receiver that is co-located to the transmitter will receive these scattered waves. The co-located receiver shares the transmitted information $\{I(n)\}$ and can use this information for the radar processing. It is assumed that the radar processing is based on one transmitted OFDM symbol described by (5) and (6) which is generated from an arbitrary information sequence $\{I(n)\}$ without any specific restrictions. A possible approach to the realization of this idea has already been investigated and proven in [4]. In the cited work it is proposed to calculate the radar range profile by simply correlating the received time domain signal $y(t)$ with the transmitted time domain signal $x(t)$

$$
\varphi_{y x}(\tau)=\int y(t) x(t-\tau) d t
$$

with $\tau$ being the time variable of the radar range profile. This approach is justified with the pulse-like structure of the time domain OFDM signal. However, the proposed approach has two major drawbacks. First, the auto-correlation function of the time domain OFDM signal is far from being ideal, which results in high sidelobes in the radar range profile. This is a severe drawback, since it drastically limits the dynamic range in scenarios with multiple scattering objects. Second, the correlation properties depend on the transmitted information $\{I(n)\}$, which makes it impossible to guarantee a reliable system performance while transmitting arbitrary information.

In order to circumvent these disadvantages, now a novel approach to OFDM radar processing will be regarded, the basic idea of which consists in using the transmitted information $\{I(n)\}$ and the received information $\left\{I_{r}(n)\right\}$ at the output of the OFDM de-multiplexer before the channel equalization and the decoding is performed. At this point the distortion from the channel is fully contained in the complex modulation symbols $\left\{I_{r}(n)\right\}$. Since all information symbols in one OFDM symbol are transmitted through the channel at different carrier frequencies separated by $\Delta f$, the received information symbols can be used in order to perform channel sensing at discrete frequencies like in stepped frequency radar. The samples of the frequency domain channel transfer function can easily be obtained by simply calculating an element-wise division

$$
I_{d i v}(n)=\frac{I_{r}(n)}{I(n)}
$$

The sampled channel impulse response, which corresponds to the radar range profile, is obtained as the inverse (discrete) Fourier transform of $\left\{I_{d i v}(n)\right\}$

$$
\begin{aligned}
& h(k)=\operatorname{IDFT}\left(\left\{I_{d i v}(n)\right\}\right) \\
& =\frac{1}{N} \sum_{n=0}^{N-1} I_{d i v}(n) \exp \left(j \frac{2 \pi}{N} n k\right), \quad k=0, \ldots, N-1
\end{aligned}
$$

As a consequence of sampling the spectrum, the resulting radar range profile is periodic in time. However, the radar range profile is not necessarily time-discrete but a continuous function in time. The continuous-time radar range profile can be calculated by a transform similar to the discrete-time Fourier transform

$$
h(t)=T \sum_{n=0}^{N-1} I_{d i v}(n) \exp \left(j \frac{2 \pi}{T} n t\right)
$$

With this processing approach all drawbacks that occur in the classic baseband processing approach described in Eq. (7) will not exist anymore. The dynamic range is only limited by the Fourier transform sidelobes, which do not cause a significant reduction of performance and can even be reduced by applying windowing functions. Also the novel processing approach is completely independent from the transmitted information, since it relates every received modulation symbol to a transmitted one. This fact guarantees a constant and reliable system performance independent from the transmitted information. The only minor disadvantage is the periodicity of the radar range profile, which can cause ambiguities in a system parameter configuration with improper subcarrier spacing.

\section{SYSTEM PARAMETERIZATION}

The most challenging part of the system design is to find one system parameterization that fits the requirements from both the radar and the communications perspective. Only in this case both applications can be operated simultaneously. Since an ideal application area for joint radar and communications platforms are intelligent transportation systems, the system parameterization that is presented here is oriented towards automotive applications. A frequency band that is suitable for both radar and communications is the 24 GHz ISM band. This band offers the additional advantage of 
license free use. Hence, an example system parameterization will be regarded for the $24 \mathrm{GHz}$ ISM band. Nevertheless, the described parameterization procedure can be adapted to any suitable band that provides sufficient radar reflectivity and tolerable attenuation for the communications application.

\section{A. Common Limitations}

Besides the individual requirements for the radar application there also exist common limitations that result from the physical properties of the channel and mainly concern Doppler tolerance and the delay between the different multipath components. In comparison to communications propagation in the case of radar propagation twice the Doppler shift occurs, which results in

$$
f_{D}=\frac{2 v_{r e l}}{\lambda}
$$

with $f_{D}$ denoting the Doppler frequency shift, $v_{r e l}$ being the relative velocity between the system platform and the scatterer. Hence, the requirement for the radar application is more critical and this requirement is considered for the system parameterization. Assuming a maximum relative velocity of $v_{\text {rel }}=200 \mathrm{~km} / \mathrm{h}$ for typical traffic scenarios this corresponds to $v_{\text {rel, } \max }=55.6 \mathrm{~m} / \mathrm{s}$ and results in a maximum Doppler shift of $f_{D, \max }=8.9 \mathrm{kHz}$. In order to not destroy the orthogonality of the subcarriers, the subcarrier spacing $\Delta f$ has to be chosen much larger than the maximum Doppler shift $f_{D, \max }$. It can be assumed that the limit $\Delta f>10 f_{D, \max }$ will definitely ensure the orthogonality of the subcarriers and even allow for relative velocities distinctly higher than $v_{\text {rel, max }}$.

The second limitation that exists for both the radar and the communications application is the maximum delay difference between the different multi-path propagation components. In order to avoid inter-symbol interference between subsequent OFDM symbols, each OFDM symbol must be extended at the transmitter with a prefix containing a partly cyclic repetition of itself with duration of $T_{P}$. This duration must correspond to the maximum time difference of the received signal components. For the radar application it must be assumed that there is a direct coupling between the transmitting and the receiving antenna on the same platform. Hence, the cyclic prefix duration must be equal to the total maximum signal travel time between platform and scatterer instead of only the time difference as for pure communication links. Again, for the radar application there is a factor of 2 involved, since the signal travels the distance between platform and scatterer twice. If we assume that due to the high attenuation of the scattering process the maximum detectable distance is $200 \mathrm{~m}$, we obtain $T_{P}>1.33 \mu \mathrm{s}$. For the communications application this corresponds to a maximum delay difference between the propagation paths of $400 \mathrm{~m}$.

\section{B. Limitations from the radar perspective}

Additional limitations that are given solely from the radar perspective mainly concern the subcarrier spacing and the OFDM symbol duration. As it has already been mentioned in
Section II, the only minor drawback of the proposed novel processing approach is the periodicity of the radar range profile. This periodicity is equal to the OFDM symbol duration, which considering that the signal travels the distance twice, results in a maximum unambiguous measurement distance of

$$
d_{\max }=\frac{c_{0}}{2 \Delta f}=\frac{T c_{0}}{2}
$$

with $c_{0}$ being the velocity of light. Hence, the OFDM symbol duration must be chosen sufficiently large in order to avoid aliasing in the radar range profile. However, considering the intended application area, this limit is absolutely not critical. Assuming again a limitation of the maximum detectable distance due to the high attenuation of the scattering process of $200 \mathrm{~m}, T>1.33 \mu \mathrm{s}$ or $\Delta f<750 \mathrm{kHz}$ is obtained.

The second constraint from the radar perspective concerns the OFDM symbol duration. In order to obtain a sufficiently high signal to noise ratio at the input of the radar processor, the energy content of the signal to be processed must be maximized. For practical applications, where the transmit power is limited, this means the integration time of the processor must be chosen as long as possible. Hence, the OFDM symbol duration must be chosen as long as possible without violating the constraints resulting from the Doppler effect.

\section{Radar Range Resolution}

One important key parameter for the performance of every radar system is the achievable range resolution. The range resolution neither depends on the employed waveform nor on the particular parameterization of the OFDM system but only on the total bandwidth occupied by the transmitted signal. The relation is as follows

$$
\Delta r=\frac{c_{0}}{2 B}=\frac{c_{0}}{2 N \Delta f}
$$

with $\Delta r$ denoting the range resolution and $B$ being the total signal bandwidth. For the intended vehicular application the resolution should be sufficiently small in order to allow for the separation of typical objects like cars, buildings, etc. It can be assumed that a range resolution in the order of 1 to $2 \mathrm{~m}$ is sufficient. This corresponds to a total signal bandwidth of approximately $100 \mathrm{MHz}$, which is also compliant with the regulations for the $24 \mathrm{GHz}$ ISM band.

\section{Final System Parameterization}

Based on the constraints regarded so far now a suitable system parameterization for an OFDM system for joint radar and communications operations will be fixed. The key parameters of an OFDM system are the subcarrier spacing $\Delta f$ and the symbol duration $T$, respectively, which are related through (2). For maximizing the received energy we have to choose $T$ as long as possible, which corresponds to choosing 
the subcarrier spacing $\Delta f$ as small as possible. Hence, we decide for the minimum spacing that is tolerable due to the Doppler constraint. In order to have round numbers $\Delta f=90.909 \mathrm{kHz}$ and $T=11 \mu \mathrm{s}$ is chosen. Also the limitation concerning the periodicity of the radar range profile is generously fulfilled with this choice. The resulting maximum unambiguous distance is $d_{\max }=1650 \mathrm{~m}$. For the length of the cyclic prefix $T_{P}=1 / 8 T=1.375 \mu \mathrm{s}$ is chosen in order to fulfill the given requirements. The total transmitted OFDM symbol duration including the cyclic prefix results in $T_{\text {sym }}=T+T_{P}=12.375 \mu \mathrm{s}$.

In OFDM systems typically powers of 2 are chosen for the number of subcarriers because this allows for efficient implementation of the necessary DFT and IDFT calculations in the transmitter and the receiver. With the already fixed parameters a bandwidth close to $100 \mathrm{MHz}$ is obtained by choosing the number of subcarriers to $N=1024$. This choice might be regarded as critical because the high number of subcarriers results in a high peak to average power ratio (PAPR) of the OFDM signal. However, there have been reported techniques in literature that allow for minimizing the PAPR by applying suitable block coding schemes to the transmitted data [5]. With the choice made for the number of subcarriers the resulting total OFDM signal bandwidth is $B=93.1 \mathrm{MHz}$, which leads to a radar range resolution of $\Delta r=1.61 \mathrm{~m}$. An overview on all system parameters is provided in Table 1.

TABLE I

SYSTEM PARAMETERS

\begin{tabular}{ccc}
\hline \hline Symbol & Quantity & Value \\
\hline$f_{c}$ & Carrier frequency & $24 \mathrm{GHz}$ \\
$N$ & Number of subcarriers & 1024 \\
$\Delta f$ & Subcarrier spacing & $90.909 \mathrm{kHz}$ \\
$T$ & Elementary symbol duration & $11 \mu \mathrm{s}$ \\
$T_{P}$ & Cyclic prefix length & $1.375 \mu \mathrm{s}$ \\
$T_{\text {sym }}$ & Transmitted symbol duration & $12.375 \mu \mathrm{s}$ \\
$B$ & Total signal bandwidth & $93.1 \mathrm{MHz}$ \\
$\Delta r$ & Radar range resolution & $1.61 \mathrm{~m}$ \\
$d_{\max }$ & Unambiguous range & $1650 \mathrm{~m}$ \\
\hline
\end{tabular}

\section{VERIFICATION SIMULATIONS}

In order to verify the operability and the performance of the proposed parameterization and processing concept a complete system model including OFDM transmitter, wave propagation, OFDM receiver and radar processing algorithms has been implemented in MatLab. In particular the intention has been to investigate the available dynamic range and resolution that is obtained when processing real OFDM signals and to assure the system operability in the presence of Doppler shift. In the following sections, first the implementation of the system model will be discussed in detail, then simulation results will be presented.

\section{A. Implementation of the System Model}

All components of the system model are implemented as individual functions in MatLab. The transmitter generates an
OFDM signal with the specified parameters from an arbitrary binary information by applying 4-PSK subcarrier modulation, arranging the modulation symbols in the frequency domain, and then calculating the sampled time domain transmit signal through an IDFT. The sampled time domain signal is then converted into a quasi-continuous signal by oversampling with a sample-and-hold element with subsequent low-pass filtering. Finally, the signal is converted to the carrier frequency in a bandpass sub-sampling representation.

The following stage is a channel model that simulates the scattering of the transmitted signal at an arbitrary number of point scatterers. For each scatterer, the time delay, the phase shift, and the attenuation are calculated individually. The attenuation is the product of the free space attenuation and the attenuation caused by the scatterer

$$
a=\sqrt{\frac{\lambda^{2} \sigma}{(4 \pi)^{3} r^{4}}}
$$

with $a$ denoting the signal attenuation in terms of voltage, $\sigma$ being the radar cross section of the scattering object, and $r$ being the distance to the scattering object. Each scatterer can be assigned an individual relative velocity, which is modeled by converting the signal to the resulting frequency (carrier plus Doppler). Then, the signal components resulting from the different scatterers are superimposed. Since the primary aim is to verify the proposed processing approach, no particular assumptions on transmitted power and antenna gain are made.

At the receiver the signal is converted back to the baseband and sampled at the modulation symbol rate. Then a DFT processing is performed in order to recover the modulation symbols in the frequency domain. Before any equalization or symbol decision is applied, the received symbols with continuous phase values are fed to the proposed radar processing algorithm described in (8) - (10). Finally, the radar range profile is calculated.

\section{B. $\quad$ Simulation Results}

In order to verify the operability and the dynamic range the processor is tested with one single point scatterer with radar cross section $\sigma=10 \mathrm{dBm}^{2}$ and relative velocity $v_{\text {rel }}=0$ at a distance of $d=30 \mathrm{~m}$. The radar range profile calculated from the received signal is plotted in Fig. 1. In order to reveal the exact shape of the response from the scatterer, the samples of the radar range profile are interpolated by a factor of 8 . This also applies to all figures that will be shown later. The scatterer is sharply depicted at a distance of $30 \mathrm{~m}$. The only sidelobes that are occurring are those resulting from the Fourier transform. Hence it has been proven that the proposed approach is working with real OFDM signals. Furthermore, an excellent sidelobe performance is obtained that exceeds the results of the classical approach described in [4] by approx. 7 $\mathrm{dB}$. By applying windowing functions in the DFT processing much higher sidelobe attenuations can be achieved.

In order to assess the capability of the proposed algorithm concerning the achievable resolution in terms of separability 


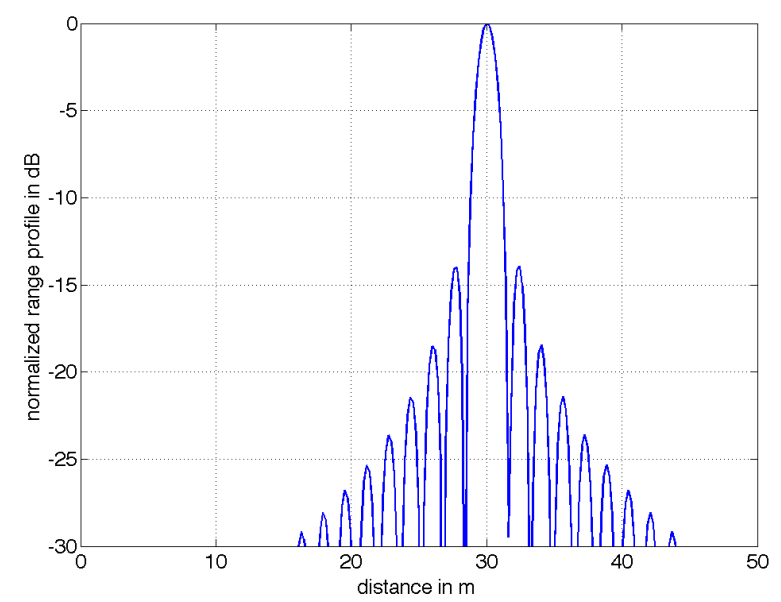

Fig. 1. Radar range profile for one single scatterer with $\sigma=10 \mathrm{dBm}^{2}$, $v_{\text {rel }}=0$, at $d=30 \mathrm{~m}$

of adjacent scatterers, a similar simulation but with two scatterers at distances $d=30 \mathrm{~m}$ and $d=31.9 \mathrm{~m}$ (again with $\sigma=10 \mathrm{dBm}^{2}$ and $v_{\text {rel }}=0$ for both objects) is performed. The relevant area of the radar range profile is shown in Fig. 2.

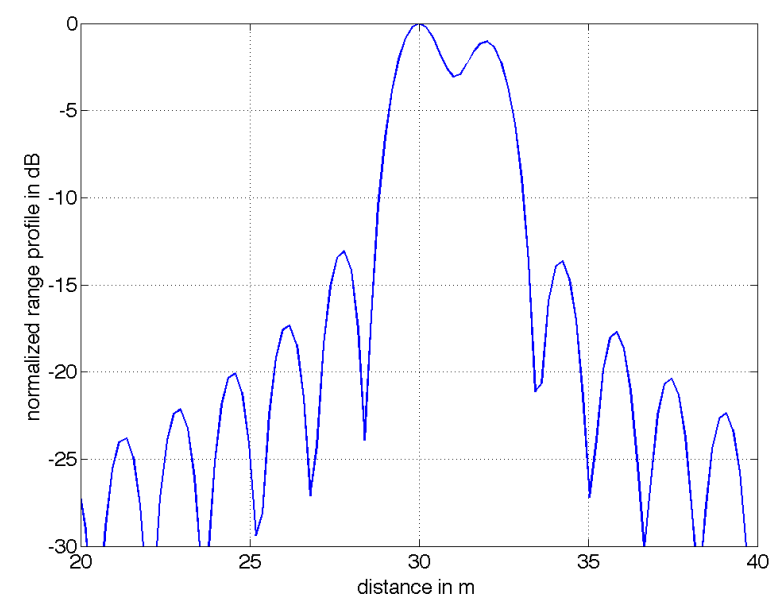

Fig. 2. Radar range profile for two scatterers with $\sigma=10 \mathrm{dBm}^{2}, v_{r e l}=0$, at $d=30 \mathrm{~m}$ and $d=31.9 \mathrm{~m}$

The drop in the radar range profile between the two scatterers amounts approximately $3 \mathrm{~dB}$. Hence, under ideal conditions two scatterers in a distance of $\Delta r=1.9 \mathrm{~m}$ are seperable, which corresponds to the usual assumption that is in practical application two targets can be resolved at twice the resolution distance of $\Delta r=1.61 \mathrm{~m}$. Also it has to be remarked that this simulation result proofs that the proposed processing approach is capable of imaging multiple objects.

Finally, which is a crucial requirement for automotive applications, the resistance of the concept and the chosen parameter set against Doppler shift has been investigated. For that purpose again the same two point scatterers have been simulated, but the second one has now been assigned a relative velocity of $v_{r e l}=100 \mathrm{~m} / \mathrm{s}=360 \mathrm{~km} / \mathrm{h}$. The relevant area of the calculated radar range profile is shown in Fig. 3. Again, the full operability of the processing algorithm is available, even

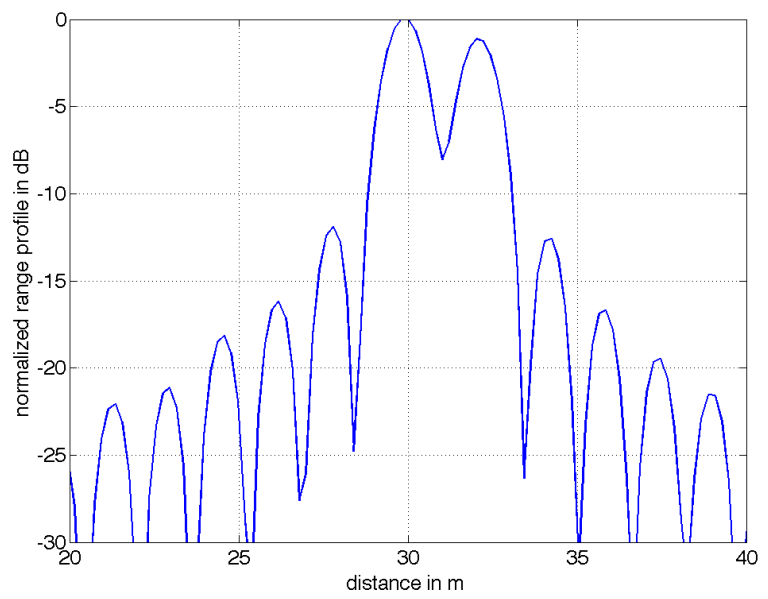

Fig. 3. Radar range profile for two scatterers with $\sigma=10 \mathrm{dBm}^{2}$, one with $v_{\text {rel }}=0$ at $d=30 \mathrm{~m}$ and the 2nd with $v_{r e l}=100 \mathrm{~m} / \mathrm{s}$ at $d=31.9 \mathrm{~m}$

though the second scatterer has even twice the speed that has been considered in the system design. Both objects are fully depicted and even better separable, only the dynamic range has slightly decreased. Hence, the Doppler shift has no influence on the distance processing. This seems to be a general advantage of OFDM radar and has already been observed in [6].

\section{CONCLUSIONS}

In this paper a detailed system design concept for a joint radar and communications system based on OFDM signals has been presented. A novel OFDM radar processing approach has been proposed, that directly operates on the modulation symbols instead of the baseband signal and overcomes the drawbacks of classic correlation based processing. A suitable system parameterization for the $24 \mathrm{GHz}$ ISM band has been derived. With MatLab simulations the operability of the proposed concept and its superior performance concerning dynamic range and resolution have been proven.

\section{REFERENCES}

[1] K. Mizui, M. Uchida, M. Nakagawa, "Vehicle-to-Vehicle Communication and Ranging System Using Spread Spectrum Technique," in Proc. IEEE Vehicular Technology Conference, pp. 335338, May 1993.

[2] N. Levanon, "Multifrequency complementary phase-coded radar signal," in IEE Proceedings Radar, Sonar and Navigation, vol. 147, no. 6, pp. 276-284, Dec. 2000.

[3] G. Lellouch, H. Nikookar, "On the capability of a radar network to support communications," in Proc. 14th IEEE Symposium on Communications and Vehicular Technology in the Benelux, pp. 1 - 5, Nov. 2007.

[4] A. Garmatyuk, J. Schuerger, Y. T. Morton, K. Binns, M. Durbin, J. Kimani, "Feasibility study of a multi-carrier dual-use imaging radar and communication system," in Proc. 37th European Microwave Conference, pp. 1473 - 1476, Munich, Oct. 2007.

[5] T. A. Wilkinson, A. E. Jones, "Minimisation of the peak to mean envelope power ratio of multicarrier transmission schemes by block coding," in Proc. IEEE 45th Vehicular Technology Conference, vol. 2, pp. $825-829$, Jul. 1995.

[6] G. E. A. Franken, H. Nikookar, P. van Genderen, "Doppler tolerance of OFDM coded Radar Signals, Proc. 3rd European Radar Conference, pp. 108 - 111, Manchester, Sept. 2006. 